\title{
A THEOREM ON THE ACCESSIBILITY OF BOUNDARY PARTS OF AN OPEN POINT SET
}

\section{EBERHARD HOPF}

Let $\Omega$ be a bounded open set in ordinary $n$-space. Denote by $p$ a point of that space. A closed subset of the boundary of $\Omega$ is called accessible from within $\Omega$ if $\Omega$ contains a semi-open Jordan curve $p(t)$, $0 \leqq t<\infty$, whose points of accumulation, for $t \rightarrow \infty$, precisely constitute that boundary part. In this note we prove the following theorem.

THEOREM 1. If $\Omega$ is a bounded open set and if $\Omega^{\prime}$ is a connected ${ }^{1}$ open subset $\Omega^{\prime}$ of $\Omega$ whose boundary has at least a point in common with the boundary of $\Omega$, then the set $C$ of all of these common boundary points contains a set accessible from within $\Omega$. If $C$ is the sum of two disjoint closed sets then each of these sets contains a set accessible from within $\Omega$.

The theorem was established and proved in order to bridge a gap in the proof of a classical theorem on surfaces $z(x, y)$ of nonpositive Gaussian curvature. Theorem $1 \mathrm{might}$, however, be of interest in other respects. The completed proof of the differential geometric theorem is given in the subsequent paper. The special case of Theorem 1 needed for the completion is that where the boundaries of the open set $\Omega$ and of the open and connected sets $\Omega^{\prime} \subset \Omega$ have only one or two points in common. None of these points need, of course, be accessible from $\Omega^{\prime}$. Theorem 1 , however, ascertains that each of these points is accessible from $\Omega$.

The conclusion of the theorem remains valid if the open set $\Omega^{\prime} \subset \Omega$, instead of being connected, is merely supposed to be connected to the vicinity of $C$. We call an open set $\Omega^{\prime}$ connected to the vicinity of a closed set $S$ if there is a neighborhood $N^{*}$ of $S$ (open set containing $S$ ) with the following property. For every neighborhood $N$ of $S$ there is a Jordan arc whose interior lies in $\Omega^{\prime}$ and whose end points lie in $N$ and on the boundary of $N^{*}$, respectively. The generalized Theorem 2 is found to be more easily proved than Theorem 1. It is convenient to extend the notion of accessibility of a closed set $S$ from an open set $\Omega$ to arbitrary closed sets (not necessarily parts of the boundary of $\Omega$ ).

Theorem 2. Let $\Omega$ and $\Omega^{\prime} \subset \Omega$ be two bounded open sets and denote by $S$ a closed set which contains all points common to the boundaries of $\Omega$

Received by the editors October 1, 1948.

1 Throughout this note the notion of connectedness is meant in the sense of arcwise connectedness. 
and $\Omega^{\prime}$. Let $\Omega^{\prime}$ be connected to the vicinity of $S$. Then $S$ contains a set accessible from within $\Omega$. If $S$ splits into two disjoint closed sets the same is true for each part.

Proof of Theorem 2. Recall the set $N^{*}$ in the definition of vicinity-connectedness. Let $N_{1}$ and $N_{2}$ be two arbitrary neighborhoods of $S$ subject to the conditions

$$
N_{1} \subset N^{*} \text {, closure of } N_{2} \subset N_{1} \text {. }
$$

Let

$$
N_{1} \Omega=\sum C,
$$

be the decomposition of the open set $N_{1}$ into disjoint connected components. The open sets

$$
C_{n}^{\prime}=C_{n} N_{2} \Omega^{\prime}
$$

are disjoint and satisfy, according as $N_{2} \subset N_{1}$ and $\Omega^{\prime} \subset \Omega$, the relation

$$
N_{2} \Omega^{\prime}=\sum C_{\nu}^{\prime} .
$$

$N_{2}$ contains $S$ and $S$ in turn contains, according to hypothesis, boundary points of $\Omega^{\prime}$. The open set $N_{\mathbf{2}}$ contains, therefore, points of $\boldsymbol{\Omega}^{\prime}$ and the sets $C_{v}^{\prime}$ cannot all be empty.

We first show that a boundary point of $C_{n}$ is either a boundary point of $\Omega$ or a boundary point of $N_{1}$. In fact, a boundary point $p$ of $C_{n}$ is a point of accumulation of $N_{1}$. If $p$ is not in $N_{1}$ it must lie on the boundary of $N_{1}$. Suppose that $p \subset N_{1}$. As a boundary point of the open set $C_{n}, p$ cannot belong to $C_{n}$. Furthermore, $p$ cannot belong to any other $C_{m}$. Otherwise a whole neighborhood of $p$ would belong to $C_{m}$ and $C_{n} C_{m}$ would not be empty since the neighborhood contains points of $C_{n} . p$ therefore is not in $\sum C_{v}=N_{1} \Omega$ and, as $p \subset N_{1}$, not in $\Omega$. On the other hand, every neighborhood of $p$ contains points of $C_{n}$, and $C_{n} \subset \Omega$. $p$ must be a boundary point of $\Omega$.

The boundary of $C_{n}$ and the boundary of the subset $C_{n}^{\prime}$ of $C_{n}$ cannot have common points except on $S$. In fact, such a point must be a boundary point of $\Omega$ since, according as $C_{n}^{\prime} C N_{2}$ and as the second inequality (1) holds, it cannot lie on the boundary of $N_{1}$. It must also be a boundary point of $\Omega^{\prime}$ since it is a point of accumulation for $C_{n}^{\prime}$ $C \Omega^{\prime} \subset \Omega$. According to the hypothesis, a common boundary point of $\Omega$ and $\Omega^{\prime}$ can only lie on $S$.

The main point of the proof is to show that among the sets $C_{n}^{\prime}$ there is at least one that is connected to the vicinity of $S$. To this purpose we first consider the "special" $C_{n}^{\prime}$, that is, those $C_{n}^{\prime}$ which have a 
boundary point, say $p_{n}$, on the boundary of $N_{2}$. We prove that the special $C_{n}^{\prime}$ must be finite in number. If there were infinitely many, the corresponding points $p_{n}$ would have a point accumulation $p^{*}$ on the boundary of $N_{2}$. Every sufficiently small neighborhood $V$ of $p^{*}$ would, according to the second inequality (1), contain no points of the boundary of $N_{1}$. We can always assume $V$ to be connected. $V$ would contain points $p_{n}, p_{m}$ belonging to two disjoint open sets $C_{n}, C_{m}$ and, therefore, all the points of a suitable Jordan arc joining them. This arc, and therefore $V$, must contain a boundary point of $C_{n}$. This point is either a boundary point of $\Omega$ or of $N_{1}$. The latter case cannot occur in $V$. Consequently, every neighborhood of $p^{*}$ contains a boundary point of $\Omega$, and, therefore, $p^{*}$ is itself a boundary point of $\Omega$. From $p_{n} \subset \Omega^{\prime}$ and $\Omega^{\prime} \subset \Omega$ it is obvious that $p^{*}$ is also a boundary point of $\Omega^{\prime}$. This is a contradiction, since a common boundary point of $\Omega$ and $\Omega^{\prime}$ must, according to the hypothesis, lie on $S \subset N_{2}$ and, therefore, cannot lie on the boundary of $N_{2}$. Hence the special $C_{n}^{\prime}$ must be finite in number. Now we make use of the hypothesis that the open set $\Omega^{\prime}$ is connected to the vicinity of $S$. For any neighborhood $N$ of $S$,

$$
N \subset N_{2}
$$

there is a Jordan arc leading from some point of the boundary of $N^{*}$ entirely through $\Omega^{\prime}$ to some point of $N$. On this arc there is, according to (1), a last point of entry into $N_{2}$. This is the initial point of a subarc which thus joins some boundary point of $N_{2}$ entirely through $N_{2} \Omega^{\prime}=\sum C_{v}^{\prime}$ to some point of $N$. Since the $C_{\nu}\left(C_{v}^{\prime} \subset C_{v}\right)$ are mutually disconnected, the subarc must lie wholly in one of the $C_{*}^{\prime}$. This must, obviously, be one of the finitely many special $C_{\nu}^{\prime}$. If we let $N$ run through a sequence of neighborhoods of $S$ closing down on $S$, and if we attach an arc and a subarc to each of these $N$, we see that one of the $C_{v}^{\prime}$, say $C_{n}^{\prime}$, must contain infinitely many of these subarcs. $C_{n}^{\prime}$ is evidently connected to the vicinity of $S$.

The result obtained is this. Given any two neighborhoods $N_{1} N_{2}$ of $S$ satisfying (1), there exist two open sets $\Omega_{1}$ and $\Omega_{1}^{\prime} \subset \Omega_{1}$,

$$
\Omega_{1} \subset \Omega, \quad \Omega_{1} \subset N_{1}, \quad \Omega_{1}^{\prime} \subset N_{2},
$$

with the following properties. The common points of the boundaries of $\Omega_{1}$ and $\Omega_{1}^{\prime}$ lie on $S . \Omega_{1}$ is connected. $\Omega_{1}^{\prime}$ is connected to the vicinity of $S .{ }^{2}$ The three sets $\Omega_{1}, \Omega_{1}^{\prime}$, and $S$ thus have precisely the properties

\footnotetext{
2 The neighborhood from whose boundary the arcs are drawn is $\mathrm{N}_{2}$. In the case of $\mathbf{\Omega}^{\prime}$ it was $N^{*}$, which could, of course, be replaced by $N_{1}$.
} 
supposed about $\Omega, \Omega^{\prime}$, and $S$ with the important additional information that $\Omega_{1}$ is connected.

We now start from a sequence of neighborhoods $N_{1}, N_{2}, N_{3}, \cdots$ of $S$ closing down on $S$. We choose them such that

$$
\text { (closure of } \left.N_{n+1}\right) \subset N_{n} \text {. }
$$

In the same way as before, starting this time from $\Omega_{1}$ and $\Omega_{1}^{\prime}$, we can construct two open sets $\Omega_{2}$ and $\Omega_{2}^{\prime} \subset \Omega_{2}$,

$$
\Omega_{2} \subset \Omega_{1}, \quad \Omega_{2} \subset N_{2}, \quad \Omega_{2}^{\prime} \subset N_{3},
$$

such that $\Omega_{2}, \Omega_{2}^{\prime}$, and $S$ have the same properties as $\Omega_{1}, \Omega_{1}^{\prime}$, and $S .{ }^{3}$ Continuing in this manner we obtain a sequence of connected open sets with the properties

$$
\Omega \supset \Omega_{1} \supset \Omega_{2} \supset \Omega_{3} \supset \cdots, \Omega_{n} \subset N_{n} .
$$

The $\Omega_{n}^{\prime}$ are no longer needed. In each $\Omega_{n}$ we choose a point $q_{n}$. As $\Omega_{n}$ is connected, $q_{n}$ and $q_{n+1}$ can be joined by an arc $\alpha_{n} \subset \Omega_{n} \subset N_{n}$. The curve $\alpha_{1}+\alpha_{2}+\alpha_{2}+\cdots$ has the desired properties, except perhaps simplicity. Every point of accumulation, as $t \rightarrow \infty$, lies in the common part of the $N_{n}$, which is $S$ since $S$ is closed. By the well known procedure of "omitting loops" we obtain a simple Jordan curve with the same properties.

The additional concluding statement of Theorem 1 is proved in the following way. Let $C=C_{1}+C_{2}$, where the parts are closed and disjoint. There exist neighborhoods $N_{1}$ of $C_{1}$ and $N_{2}$ of $C_{2}$ without common points. The boundaries of the sets $\Omega+N_{2}$ and $\Omega^{\prime}$ obviously have precisely $C_{1}$ as their common part. According to Theorem 1, $C_{1}$ is therefore accessible from $\Omega+N_{2}$. The Jordan curve in $\Omega+N_{2}$ that converges towards $C_{1}$ must, from a certain point on, lie in $N_{1}$ and thus outside of $N_{2}$, in other words in $\Omega . C_{1}$ is, therefore, accessible from $\Omega$.

NeW YoRK UnIVERSITY

${ }^{3} N^{2}$ is the neighborhood from whose boundary the arcs are drawn through $\Omega_{2}$ to the vicinity of $S$. 